\title{
Assessment and Correlation of Uric Acid Concentration in Saliva and Serum of Patients Attending Tertiary Care Centre
}

\author{
Surakchhya Gautam,' Sangita Thapa,' Anju Khapung ${ }^{2}$ \\ 'Department of Biochemistry, Kathmandu Medical College and Teaching Hospital, Duwakot, Kathmandu, Nepal, ${ }^{2}$ Department \\ of Community Dentistry, College of Dental Sciences, Nepal Medical College, Jorpati, Nepal.
}

\begin{abstract}
Introduction

Serum uric acid (UA) is associated with many health conditions including renal, cardiovascular and metabolic disorders. Diagnosis and monitoring often require painful invasive procedures which will add undue stress to the patients. The aim of this study was to correlate salivary and serum UA, so that, salivary sampling will help to bypass invasive measures and evaluate the condition of both healthy and the diseased.
\end{abstract}

\section{Methods}

This hospital based analytical cross sectional study was conducted in 100 participants between age groups 21 to 82 years. Demographic data including height, weight and blood pressure were recorded. Serum and salivary samples were collected and UA level in both type of samples were estimated and correlation statistics was carried out.

\section{Results}

Total participants were categorized in three groups, 20-40, 41-60 and more than 60 years, among which maximum participants 43 (43\%) were between 41-60 years of age. Mean serum and salivary UA (mg/dl) were 4.15 and 1.90 respectively, exhibiting moderate positive correlation $(\mathrm{r}=0.338)$. They were correlated with significant "p-value" according to gender (male, $r=0.429, p=0.006$, female, $r=0.277, p=0.03)$, age groups (20-40yrs, $r=0.383, p=0.03$ and 41-60yrs, $r=0.412, p=0.006$ ) and disease conditions $(\mathrm{r}=0.338, \mathrm{p}=0.006)$.

\section{Conclusions}

Salivary and serum UA were associated in our study so salivary UA can be used as a biomarker. Salivary UA was found to be more gender specific and can also be used as a screening tool for young and adult population.

Key words: hyperuricemia; saliva; serum; uric acid.

Correspondance: Dr. Surakchhya Gautam, Department of Biochemistry, Kathmandu Medical College and Teaching Hospital, Sinamangal, Kathmandu, Nepal. Email: gautamsurakshya8758@gmail.com. Phone: +977-9840553006. 


\section{INTRODUCTION}

Uric acid (UA) has its anti- and pro- oxidant effects on a wide range of peripheral tissues. The derangement of systemic UA level has been associated with disease complex like hypertension, chronic kidney diseases and metabolic syndrome. ${ }^{1.2}$

Saliva is an ultra- filtrate of blood which is evolving into a popular noninvasive diagnostic tool. ${ }^{3,4}$ A robust positive serum- saliva UA correlation has promoted the utility of salivary uric acid as an important biomarker for indexing health and disease risk. ${ }^{1,5}$

Patient compliance, non-invasiveness, economical and ease of collection methods are considered as advantages of salivary sampling over serum sampling. ${ }^{1}$ The process of screening a large group of samples also gets considerably simplified when a noninvasive salivary sampling is utilized for the purpose. ${ }^{6}$ This study aimed to explore the possible linear correlation between serum and salivary uric acid and its usefulness as a biomarker for monitoring different clinical conditions.

\section{METHODS}

An analytical cross sectional study was conducted in the Department of Biochemistry, KMCTH, Duwakot, which is a tertiary care center and receives a variety of clinical cases. The study was conducted from $21^{\text {st }}$ May 2019 to $31^{\text {st }}$ August 2019 after the approval from Institutional Review Committee (Ref: 20052019115). The participants of the study were selected through convenience sampling from patients who had provided their serum samples for routine or specific health checkups to the laboratory of KMCTH, Duwakot. Sample size of 100 was calculated using the formula for estimating population proportion and the reference values were obtained from a study by Shibasaki et al. ${ }^{7}$
An informed written consent was obtained from all the participants of the study and data collection procedure was then carried out. Participants with conditions known to affect salivation including Sjogren's syndrome, autoimmune diseases and pregnancy were excluded. Demographic data including age, sex, body weight, heights were obtained on the proforma. Blood pressure was measured with the participant sitting on a chair for a minimum of three minutes in a resting and relaxed state. Blood pressure was measured in both arms and the higher value thus obtained was considered as the actual blood pressure of the individual. A person is considered to have high blood pressure if the systolic value is over $140 \mathrm{~mm}$ of $\mathrm{Hg}$, the diastolic value is over $90 \mathrm{~mm}$ of $\mathrm{Hg}$, or both.

Participants were categorized as healthy and diseased group. Disease conditions that were noted in the participants were diabetes mellitus, hypertension and thyroid disorder. The weight of the participants was taken using a digital weighing machine (OEM, QF-2003A) and height of the participants was measured using the measuring scale (Height measuring scale 110805433). The height and weight values thus obtained were utilized to calculate the BMI of the individual as, $\mathrm{BMI}=$ weight in $\mathrm{kg} /$ (height in meter). ${ }^{2}$

Salivary sample was collected from the participants in the morning. Stimulated saliva was obtained by asking the participants to chew on rolled cotton for two minutes; the salivary sample thus obtained was placed in a five $\mathrm{ml}$ syringe and was then used for the measurement of uric acid concentration in saliva. The sample was compressed and stored at $-20^{\circ} \mathrm{C}$ until it was assayed. At the time of assessment of salivary sample, it was centrifuged at $3000 \mathrm{rpm}$ for 10 min, followed by collection of the supernatant (about $2 \mathrm{ml}){ }^{7}$ 
On the same day of saliva collection, five $\mathrm{ml}$ of participant's blood was also collected to examine the uric acid concentration in serum. Under aseptic condition, blood sample was obtained by venipuncture from the medial cubital vein in a capped tube. Analysis of both serum and salivary uric acid was carried out using Uricase method of estimation using Humalyzer Primus Version 3.2e semiautoanalyzer.

\section{Statistical analysis}

Data was entered in Microsoft excel 2007 version and transferred to Statistical Package for Social Sciences (SPSS) version 16.0 for required analysis. Descriptive and summary statistics like percentage, mean and standard deviation were calculated. The data for serum uric acid and salivary uric acid were found to be normally distributed using the normality test. So the data were analyzed using parametric tests. Correlation between serum and salivary uric acid was analyzed using Pearson Correlation. Salivary uric acid and serum uric acid were also correlated among the different age groups, gender and disease conditions by Pearson Correlation.

\section{RESULTS}

The mean age of the 100 participants of the study was 49.64 years among which 40 (40\%) were male and $60(60 \%)$ were female. The participants were categorized into three groups according to the age groups, 20-40 years, 41-60 years and $>60$ years. The majority of the participants of the study $43(43 \%)$ were between 41-60 years of age, while 32 (32\%) participants were from the 20-40 years age group and $25(25 \%)$ participants were above 60 years of age.

The average BMI of participants was found to be 24.46 (standard deviation (S.D) 3.34), among which 4 (4\%) of the participants were underweight, 50 (50\%) were within the normal range, whereas $41(41 \%)$ were found to be overweight and $5(5 \%)$ obese. In the current study, 34 (34\%) of the participants reported to have a preexisting medical condition including Diabetes mellitus, hypertension and thyroid disorder while rest of the 66 $(66 \%)$ reported not having any comorbidities. Other characteristics and descriptive statistics of the participants including age, gender, serum uric acid, salivary uric acid, blood pressure and BMI has been presented in table 1.

Table 1. Characteristics of study populations and biomarker levels.

\begin{tabular}{|c|c|c|c|c|c|c|}
\hline \multirow{2}{*}{ Variables } & \multirow{2}{*}{ Minimum } & \multirow{2}{*}{ Maximum } & \multirow{2}{*}{ Mean } & \multirow{2}{*}{$\begin{array}{c}\text { Standard } \\
\text { Deviation } \\
\text { (S.D) }\end{array}$} & \multicolumn{2}{|c|}{$\begin{array}{c}\text { Confidence } \\
\text { interval }\end{array}$} \\
\hline & & & & & $\begin{array}{l}\text { Upper } \\
\text { bound }\end{array}$ & $\begin{array}{l}\text { Lower } \\
\text { bound }\end{array}$ \\
\hline 1. Age (years) & 21 & 82 & 49.64 & 15.9 & 46.49 & 52.79 \\
\hline 2. Serum uric acid (mg/dl) & 0.69 & 9.66 & 4.15 & 2.03 & 3.74 & 4.55 \\
\hline 3. Salivary uric acid (mg/dl) & 0.06 & 9.43 & 1.90 & 1.85 & 1.53 & 2.27 \\
\hline 4. Systolic blood pressure $(\mathrm{mm} \mathrm{Hg})$ & 100 & 180 & 130.90 & 15.93 & 127.74 & 134.06 \\
\hline 5. Diastolic blood pressure $(\mathrm{mm} \mathrm{Hg})$ & 70 & 180 & 84.20 & 13.63 & 81.5 & 86.9 \\
\hline 6. Height $(\mathrm{m})$ & 1.25 & 1.83 & 1.60 & 0.11 & 1.58 & 1.62 \\
\hline 7. Weight $(\mathrm{Kg})$ & 42 & 87 & 62.59 & 9.95 & 60.62 & 64.56 \\
\hline 8. Body Mass Index $\left(\mathrm{kg} / \mathrm{m}^{2}\right)$ & 17.56 & 32.50 & 24.46 & 3.34 & 23.79 & 25.12 \\
\hline
\end{tabular}

$\mathrm{N}=100$ 
In the current study, the average level of serum uric acid (mg/dl) was 4.15 ranging from 0.69 to 9.66 while the average level of salivary uric acid (mg/dl) was 1.90 ranging from 0.06 to 9.43. In the current study mean height $(\mathrm{m})$ was 1.60 and mean body weight $(\mathrm{kg})$ was 62.59 . The average BMI thus, was calculated to be $24.46 \mathrm{~kg} / \mathrm{m}^{2}$.

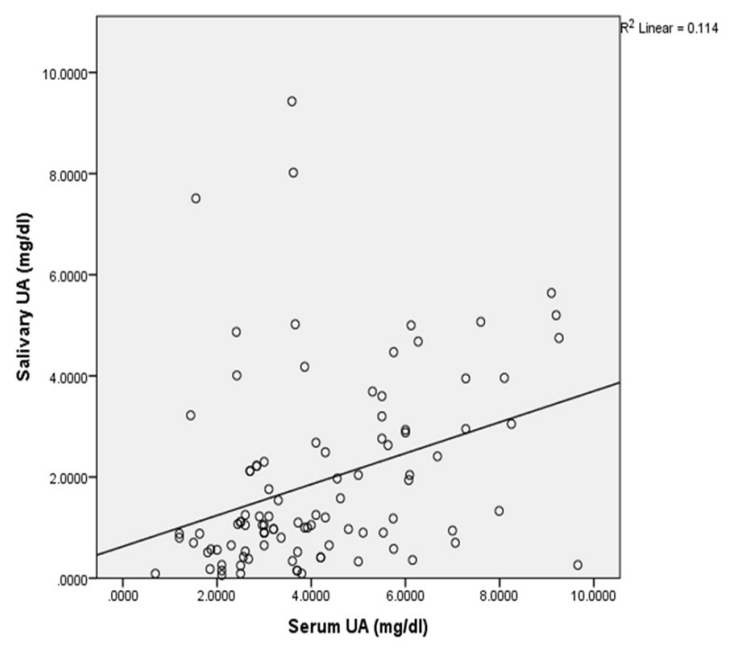

Figure 1. Scatter plot showing correlation between serum uric acid and salivary uric acid in study participants. $\left(n=100, R^{2}=0.114, r=0.338\right.$, $p$-value $=0.001 *$ )

$p$-value $<0.05$ statistically significant $*$ UA $=$ Uric Acid

As shown in the scatter plotfigure 1, concentration of salivary and serum uric acid were positively correlated. Correlation is significant at 0.01 level $(\mathrm{p}=0.001)$. Correlation is of moderate type $(\mathrm{r}=$ $0.338)$.

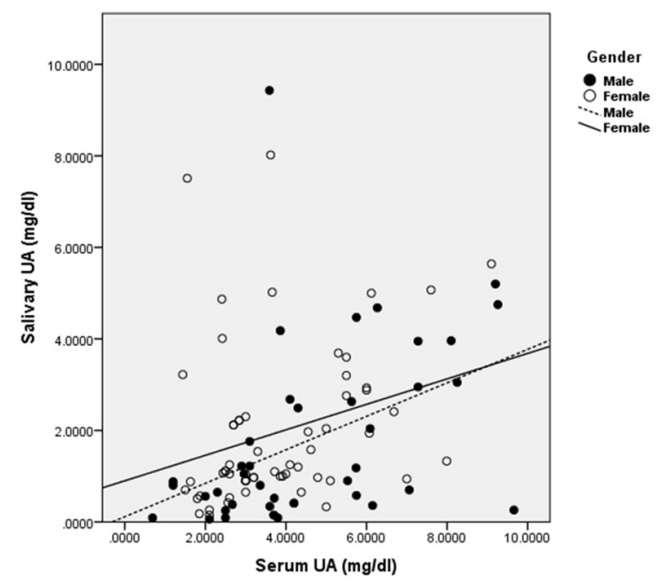

Figure 2. Scatter plot of salivary and serum concentration of uric acid in study participants according to gender (For male $R^{2}$ Linear $=0.184$, $r=0.429, p$-value $=0.006^{*}$. For female $R^{2}$ Linear $=0.077, r=0.277$, $\mathrm{p}$-value $=0.03^{*}$ )

p-value $<0.05$ statistically significant* UA $=$ Uric Acid
As shown in figure 2, the concentration of salivary and serum uric acid in the study participants were positively correlated according to the gender with statistically significant $p$ value $(p=0.006$ for male and $p=$ 0.03 for female). But the correlation in this case was found to be moderate in male participants and weak in female participants according to Pearson's correlation coefficient $(r=0.429$ for male and $r=0.277$ for female).

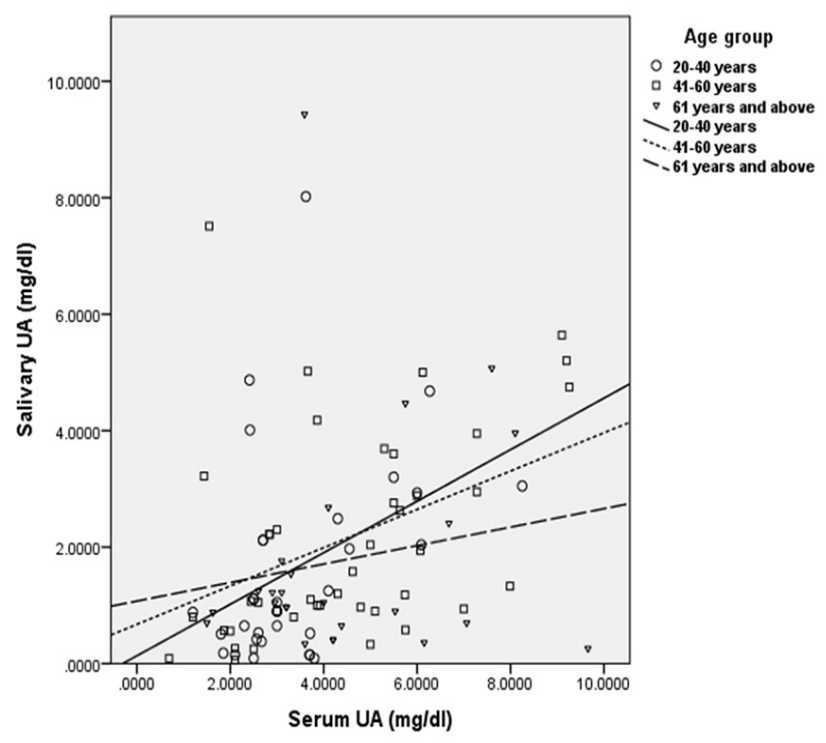

Figure 3. Scatter plot of salivary and serum concentration of uric acid in study participants according to age-group (For 20-40 years, $R^{2}$ Linear $=0.146, r=0.383, p$-value $=0.03^{*}$, for 41 60 years, $R^{2}$ Linear $=0.169, r=0.412, p$-value $=0.006^{*}$, for 61 years and above, $R^{2}$ Linear $=0.026, r=0.160, p$-value $=$ $0.446 *)$

p-value $<0.05$ statistically significant $*$ UA $=$ Uric Acid

As shown in the figure 3, positive correlation was seen between concentration of salivary and serum uric acid in age group of 20-40 years $(p=0.03)$ and $41-60$ years $(p=0.066)$. The correlation is moderate among the age group of $41-60$ years $(r=0.412)$ and $20-40$ years $(r=$ 0.383). But weak correlation is seen among elderly population above the age group of more than 60 years. 


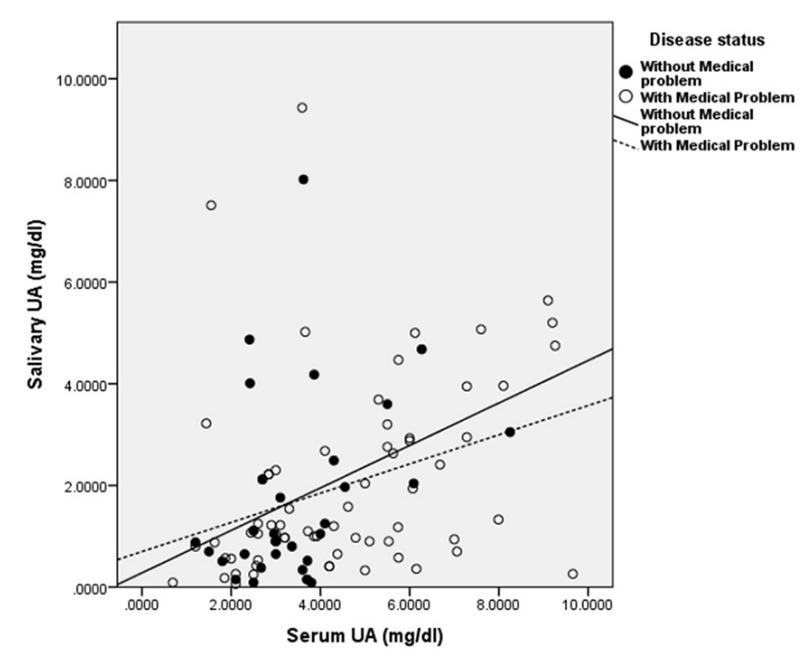

Figure 4. Scatter plot of salivary and serum concentration of uric acid in study participants according to disease condition (For those with disease, $R^{2}$ Linear $=0.112, r=0.338, p$-value $=0.05$, for those without disease, $\mathrm{R}^{2}$ Linear $=0.114, \mathrm{r}=0.334$, $\mathrm{p}$-value $=0.006 *$ )

p-value $<0.05$ statistically significant $*$ UA $=$ Uric Acid

As shown in the figure 4, positive correlation was seen in concentration of salivary and serum uric acid according to disease conditions $(p=0.05)$ as well as for those without disease condition ( $p$ $=0.006)$. Correlation is moderate and correlation is significant only for those without disease $(\mathrm{p}=$ 0.006 and $\mathrm{r}=0.334$ ).

\section{DISCUSSION}

The advantages of using saliva for diagnosis compared to other biological spectrum are the easy availability, simple and non- invasive collection that can be done by individual themselves, and easy, low cost storage. In addition to these advantages, saliva collection offers a painless alternative, eliminating the stress that the patient can feel, which can be useful for geriatric, pediatric, obese, patients with mental deficiency, prisoners etc. ${ }^{8}$

The solid components of saliva include organic and inorganic molecules which are found dissolved in the aqueous components. The inorganic part is composed of weak and strong ions like $\mathrm{Na}^{+}, \mathrm{K}^{+}, \mathrm{Cl}^{-}, \mathrm{Ca}^{2+}, \mathrm{HCO}_{3^{\prime}} \mathrm{Mg}^{2+}, \mathrm{NH}_{3}$ etc. and the organic parts comprise of components such as body secretions products like urea, uric acid, creatinine, putrefaction products and more than 400 types of proteins. ${ }^{8}$ Several types of inflammatory biomarkers like interleukins and tumor necrosis factor which are associated with both oral as well as systemic diseases have been detected in saliva in different studies. ${ }^{9}$ As it is an ultra-filtrate of plasma, saliva may be used for diagnosing systemic diseases like diabetes mellitus, cardiovascular diseases, viral infection, pancreatic cancer, breast cancer etc. by establishing linear correlation between serum and salivary levels of important biomarkers. ${ }^{10}$ As mentioned by Jenna L Riis, only a thin layer of epithelial cells separates the salivary ducts from the systemic circulation which makes ultrafiltration and exchange of molecules possible between serum and saliva, our study, thus, ventured to explore the possibility of positive correlation between serum and salivary uric acid levels. ${ }^{1,8}$

In our study, concentration of salivary and serum uric acid were positively correlated and correlation was found to be significant at 0.01 level ( $p=0.001, r=0.338)$. This finding is similar to that of the study done by Jenna L Riis et al., where they found value of salivary uric acid to be stable while assessing the validity and stability of salivary uric acid levels among 99 healthy young adults. ${ }^{1}$ Our study uses a sample of healthy young adults and we have observed the association, strength and nature of correlation between serum and salivary uric acid. A significant positive correlation between salivary and serum uric acid has also been reported by Koichi Shibasaki et al. ${ }^{7}$ Urmila Singh et al have also reported a similar finding of positive linear correlation between salivary and serum uric acid levels in pregnant females which may pave a breakthrough for noninvasive assessment in pregnant females. ${ }^{11}$

Eduardo Ottobell Chielle et al in the year 2000 also reported a significant correlation between salivary and serum uric acid among 149 participants. ${ }^{12}$ In the study done by Giancarlo Bilancio, the saliva and serum uric acid level were different but correlated, which is a similar finding to our study. They have also reported a positive correlation with other salivary markers like creatinine and potassium which highlights the scope of further studies in this field. ${ }^{13}$ In our study salivary levels 
of uric acid values were lower than serum uric acid levels, as reported by Naresh Yajamanam et al. $^{14}$ The lower salivary levels of important biomarkers may be attributable to the common understanding that salivary components are ultra filtrates of plasma and thus their values are likely to be smaller than that compared to plasma.

Honggang Wang et al. found a positive correlation between BMI and serum uric acid among 39,736 participants. $^{15}$ The study done by Aisin $\mathrm{D}$ Martinez et al, demonstrates that increased salivary uric acid levels were related to higher BMI percentiles in females of all ages. It shows that BMI has a larger effect on increasing salivary uric acid levels among younger girls and adult women. ${ }^{16}$ Hui Zhou et al, however, found a stronger positive BMI and salivary uric acid association for both men and women with a stronger association for men than women. ${ }^{17}$ Our study also exhibited a moderate positive correlation of serum and salivary uric acid in males $(p=0.006, r=0.429)$ and weak correlation in females $(p=0.03 . r=0.277)$ with " $p$ value" being statistically significant. A high BMI (overweight and obese) is considered as a risk factor of hyperuricemia and altogether $46 \%$ of our study population presented with a high BMI. However, a significant positive correlation between serum and salivary uric acid levels and BMI could not be established. This result observed in our study needs further research in a larger sample size to establish or refute this linear correlation.

Understanding the relationship between each diseaseand serum uricacid hasbeenimportantdue to the potential benefit which could be achieved by applying it to new treatment strategies. Feig et al mentioned that early hypertension developed in children and adolescents with hyperuricemia could be reversed with urate reduction. ${ }^{18}$ Different studies enlightened upon the relationship between hyperuricemia and different disease like gout, nephrolithiasis, heart failure, insulin resistance, metabolic syndrome, atherosclerosis, endothelial dysfunction, acute chronic renal impairment and hypertension. ${ }^{19}$ In our study, a significant linear correlation was observed between salivary and serum uric acid levels in participants with comorbidities like Diabetes Mellitus, Hypertension and Thyroid disorders ( $p=0.05, r=0.338)$. Maria Soukup et al. have reported that salivary uric acid was significantly increased in patient with metabolic syndrome and they have suggested that salivary uric acid may be a useful biomarker for noninvasive monitoring of cardiometabolic risk. ${ }^{20}$ In the study done by Malensz Maciejczyk, the uric acid concentration in stimulated whole saliva correlated positively with systolic and diastolic blood pressure and uric acid content in plasma which is a similar finding to that of our study. Like our study, they have also used samples from stimulated saliva in their study. ${ }^{21}$ In the study done by Jacopo Troisi et al, serum and salivary uric acid and insulin levels were significantly correlated, however their values tended to be only slightly higher in the obese patients. ${ }^{22}$

In our study, positive correlation was seen between concentration of salivary and serum uric acid in age group of $20-40$ years $(p=0.03)$ and 41 60 years $(p=0.006)$. The correlation is moderate among the age group of 41-60 years $(r=0.412)$ and $20-40$ years $(r=0.383)$. But weak correlation is seen among elderly population above the age group of more than 60 years $(r=0.160)$. This may implicate the utility of salivary uric acid in young and adult population as a screening test for hyperuricemia. D Conen et al, have also reported a significant association of serum uric acid level with age. ${ }^{23}$

Moderate correlation between serum and salivary UA levels $(r=0.383)$ in young patients compared to older ones implicate that salivary uric acid can be utilized for disease detection as well as for screening test in younger population. As studies on a larger sample size may provide a better clarity to the results obtained in this study, further well designed, large scale, longitudinal studies may be able to confirm or refute our findings. As our extensive literature search could not yield any positive results, this study may be one of the pioneering studies if not the first in the field of salivary diagnostics in general and salivary uric 
acid in particular in Nepal.

We ventured into a nascent area of research in the context of Nepal through our study which is likely to attract the interest of fellow researchers who may pursue the interesting and uncharted territories of salivary diagnostics. A clearer result may have been obtained with multi-centric studies involving a large sample size, which can thus be considered a limitation of the present study.

\section{CONCLUSION}

A linear correlation was observed between serum and salivary values of uric acid which opens an interesting avenue for non invasive sampling for health and disease detection. We hope the findings of this study paves way for further research in the field of salivary diagnostics in Nepal.

\section{ACKNOWLEDGEMENTS}

The authors would like to acknowledge the contribution of the Department of Biochemistry, KMCTH, Laboratory, Duwakot during sample processing. The authors would also like to acknowledge the contribution of Dr. Galav Adhikari in the preparation of this manuscript.

Conflict of interest

Nil

6. Pandya D, Nagrajappa AK, Ravi KS. Assessment and correlation of urea and creatinine levels in saliva and serum of patients with chronic kidney disease, diabetes and hypertension- A research study. J Clin Diagn. 2016; 10(10); Zc58Zc62. DOI: 10.7860/JCDR/2016/20294-8651.

2. Khosla UM, Zharikov S, Finch JL, Nakagawa T, Roncal C, Mu W, et al. Hyperuricemia induces endothelial dysfunction. Kidney Int. 2005; 67; 17391742.

3. Nagarathinam AE, Kumar TD, Kumar AR, Vasanthira K, Lakshmi RS, Jayant VS. Salivary urea and creatinine as a diagnostic marker of chronic disease- Review. IOSR- JDMS. 2017; 16(4); 95-100 DOI: 10.9790/0853-16040995100

4. Devraj SD. Salivary Biomarkers- A Review. J Pharm Sci and Res. 2013; 5(10); 210-212.

5. Fatima G, Uppin RB, Kasagani S, Tapshetty R, Rao A. Comparision of Salivary Uric Acid Level among Healthy Individuals without Periodontitis with that of smokers and Non- smokers with Periodontitis. J Adv Oral Res. 2016; 7; 1 DOI: 10.2047/ joaor-07-01-024
7. Shibasaki K, Kimura M, Ikareshi R, Yamaguchi A, Watanabe T. Uric acid concentration in saliva and its changes with the patients receiving treatment for hyperuricemia. Metabolomics. 2012; 8; 484491. DOI; 10.1007/S11306-011-0334-Z

8. Lima DP, Diniz DG, Moimaz SAS, Sumida $\mathrm{DH}$, Okamoto AC. Saliva: reflection of the body. Int J Infect Dis. 2010; 19; e184-e188. DOI: 10.1016/j-ijid.2009.04.022

9. Rathnayake N, Akerman S, Klinge B, Lundegren N, Jansson H, Tryselius $\mathrm{Y}$, et al. Salivary Biomarkers for detection of Systemic Diseases. PLOS ONE. 2013; 8(4); e61356.

10. Zhang CZ, Cheg XQ, Li JY, Zhang P, Yi $\mathrm{P}, \mathrm{Xu} X$, et al. Saliva in the diagnosis od diseases. Int J Oral Sci. 2016; 8; 133-137.

11. Singh U, Solanki V, Mehrotra S, Sharma R. 
Evaluation of Applicability of Salivary Uric Acid measurement in preeclampsia and Normal pregnancy and its correlation with serum Uric Acid. Te J Obstet Gynaecol India. 2019; 69(1); 62-68.DOI: 10.1007/ S13224-018-1124-6

12. Chielle EO, Casarin JN. Evaluation of salivary oxidative parameters in overweight and obese young adults. Arch Endocrinol Metab. 2017; 61(2); 152.DOI: 10.1590/2359-3997000000227.

13. Bilancio G, Cavallo P, Lombardi C, Guarino E, Cozza V, Giordano F, et al. Saliva for assessing creatinine, uric acid and potassium in nephropathic patients. BMC Nephrology. 2019; 20; 242. DOI: 10.1186/S12882-019-1437-4

14. Yajamanam N, Vinapamula KS, V S, Bitla AR, Rao PVLNS. Utility of saliva as a sample to assess Renal function and Estimated Glomerular Filtration Rate. Saudi J Kidney Dis Transpl. 2016; 27(2); 312-319.

15. Wang H, Wang L, Xie R, Dai W, Gao C, Shen $P$, et al. Association of serum uric acid with Body Mass Index: A Cross- Sectional study from Jiangsu Province, China. Iranian J Pub Health. 2014; 43(11); 1503-1508.

16. Martinez AD, Ruelas L, Granger DA. Association between body mass index and salivary uric acid among Mexican -origin infants, youth and adults: Gender and developmental differences. Dev Psychobiol. 2017; 59(2); 225-234 DOI: 10.1002/dev.21492

17. Zhou H, Liu Z, Chao Z, Chao Y, Ma L, Chen $X$, et al.Nonlinear relationship between serum uric acid body mass index: a crosssectional study of a general population in coastal China. J Transi Med. 2019; 17; 389. DOI: 10.1186/S12967-019-02142-9

18. Feig DI, Soietsky B, N R, Johnson RJ. Effects of Allopurinol on Blood pressure of Adolescents with Newly Diagnosed Essential Hypertention: A Randomized Trial. JAMA. 2008; 300(8); 924-932. DOI: 10.1001/jama.300.8.924

19. Stewart DJ, LangloIs V, Noone D. Hyperuricemia and Hypertension: Links and Risks. Integrated Blood Pressure Control. 2019; 12; 43-62.

20. Soukup M, Biesiada I, Henderson A, Idowu B, Rodeback D, Ridpath L, et al. Salivary uric acid as a noninvasive biomarker of metabolic syndrome. Diabetology and Metabolic Syndrome. 2012; 4; 14.

21. Maciejczyk M, Janusz KT, Wasilewska UA, Kossakowska A, Zalewska A. A case control study ofSalivary Redox Homeostasis in Hypertensive children. Can Salivary Uric Acid be a marker of Hypertension? J Clin Med. 2020; 9; 837. DOI: 10.3390/jcm9030837

22. Troisi J, Belmonte F, Bisogno A, Lausi O, Marciano F, Cavalla P, et al. Salivary markers of hepatometabolic comorbidities in pediatric obesity. Digestive and Liver Disease. 2019; 51(4); 516-523. DOI: 10.1016/j. dld.2018.11.009

23. Conen D, Wierlisbach V, Bovet P, Shamlaye C, Riesen W, Paccaud F, et al. Prevalence of hyperuricemia and relation of serum uric acid with cardiovascular risk factors in a developing country. BMC Public Health. 2004; 4 ; 9 .

Citation: Gautam S, Thapa S, Khapung A. Assessment and Correlation of Uric Acid Concentration in Saliva and Serum of Patients Attending Tertiary Care Centre. JCMS Nepal. 2020; 16(4):222-29. 